\title{
Erratum to: PGRMC1 contributes to doxorubicin-induced chemoresistance in MES-SA uterine sarcoma
}

\author{
Szu-Ting Lin • Eugenie Wong Soon May • Jo-Fan Chang • \\ Ren-Yu Hu $\cdot$ Lily Hui-Ching Wang $\cdot$ Hong-Lin Chan
}

Published online: 17 February 2015

(C) Springer Basel 2015

Erratum to: Cell. Mol. Life Sci.

DOI 10.1007/s00018-014-1831-9

The original publication of the article unfortunately contained an error in Fig. 2. The correct figure is given below.

The online version of the original article can be found under doi:10.1007/s00018-014-1831-9.

S.-T. Lin · R.-Y. Hu

Institute of Bioinformatics and Structural Biology, National

Tsing Hua University, No. 101, Kuang-Fu Rd. Sec. 2, Hsin-chu

30013, Taiwan

E. W. S. May · J.-F. Chang

Department of Life Science, National Tsing Hua University, No.

101, Kuang-Fu Rd. Sec. 2, Hsin-chu 30013, Taiwan

L. H.-C. Wang

Institute of Molecular and Cellular Biology and Department of

Medical Science, National Tsing Hua University, No. 101,

Kuang-Fu Rd. Sec. 2, Hsinchu 30013, Taiwan

H.-L. Chan $(\bowtie)$

Institute of Bioinformatics and Structural Biology and

Department of Medical Sciences, National Tsing Hua

University, No. 101, Kuang-Fu Rd. Sec. 2, Hsin-chu 30013,

Taiwan

e-mail: hlchan@mx.nthu.edu.tw 

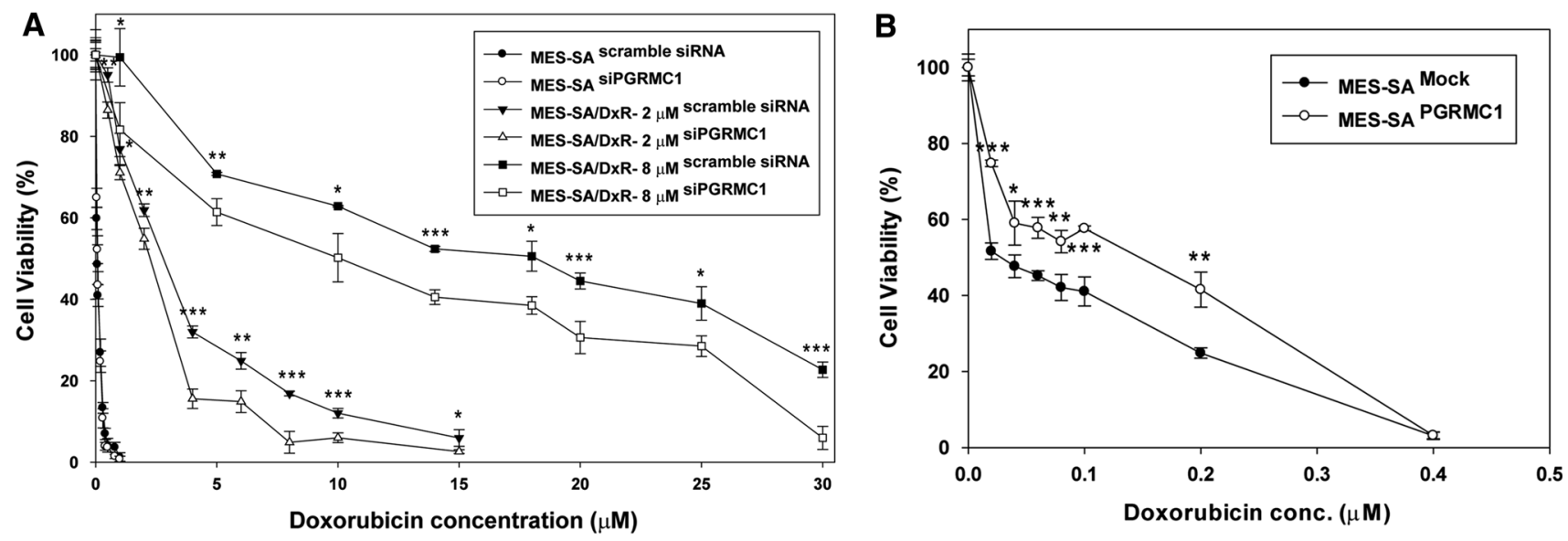

C
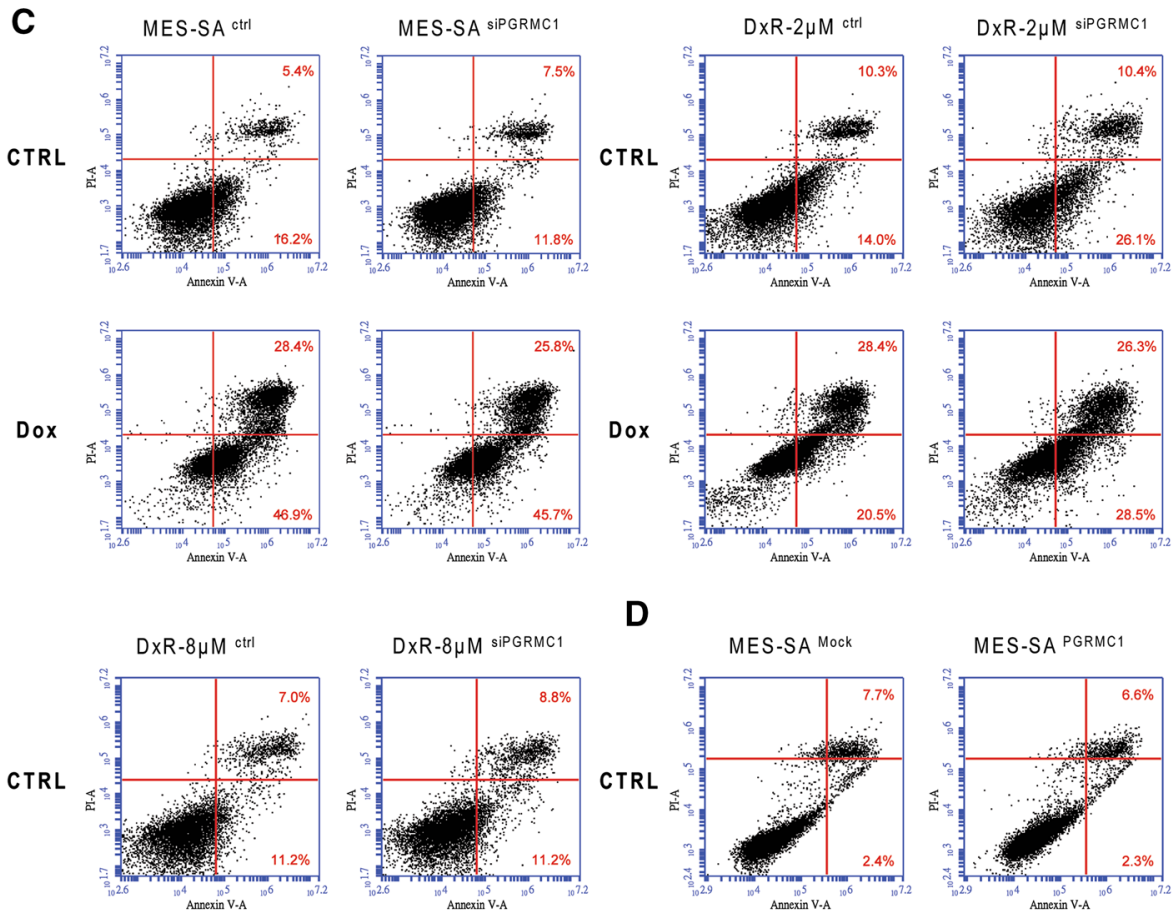

DXR-8uM SiPGRMC1

D
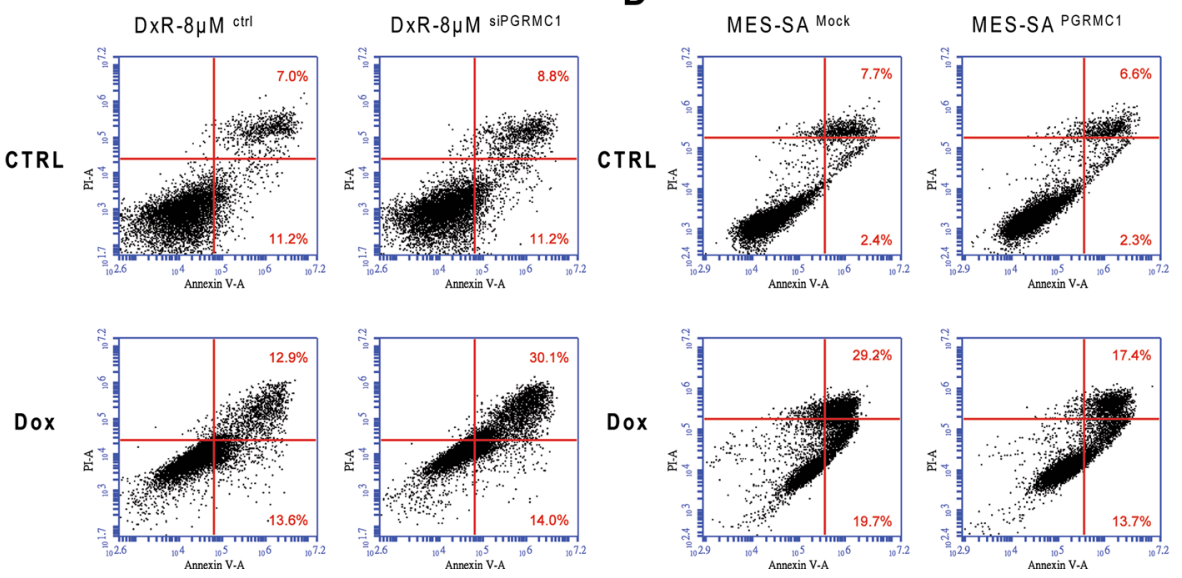


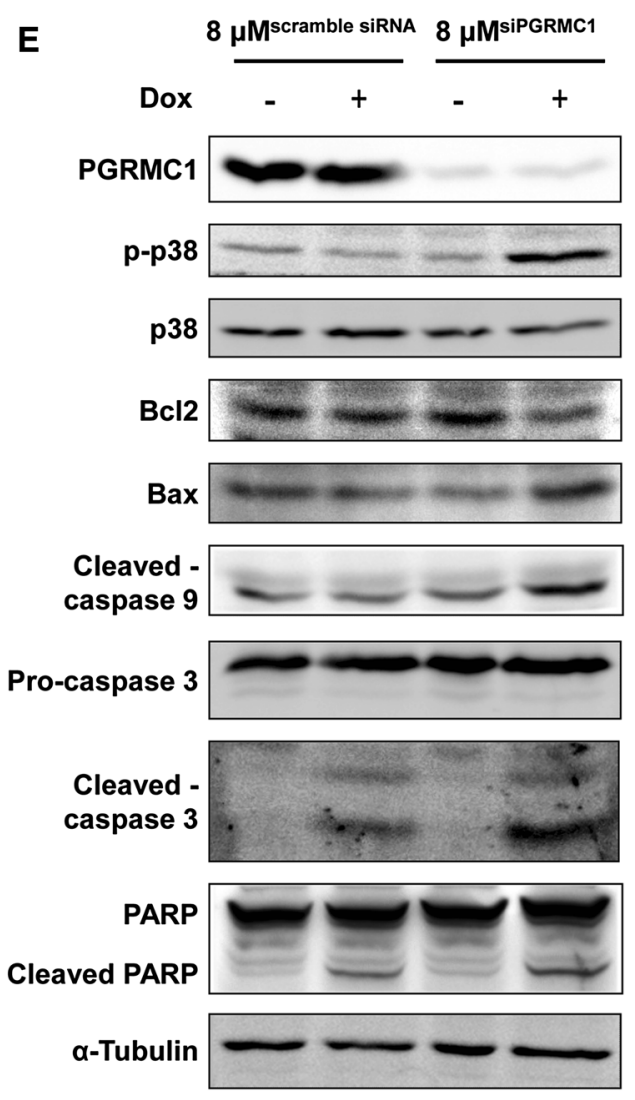

Fig. 2 Effects of PGRMC1 knockdown and PGRMC1 overexpression on cell viability, apoptosis, and level of apoptotic factors in resistant (MES-SA/DxR-2 $\mu \mathrm{M}$ and MES-SA/DxR-8 $\mu \mathrm{M}$ ) and sensitive (MES-SA) uterine sarcoma cells. a Effect of PGRMC1 knockdown on the viability of MES-SA, MES-SA/DxR-2 $\mu \mathrm{M}$, and MES-SA/DxR- $8 \mu \mathrm{M}$ cells treated with doxorubicin in a dosedependent manner. MTT-based viability assays were performed in which 7000 MES-SA, MES-SA/DxR-2 $\mu \mathrm{M}$, and MES-SA/DxR-8 $\mu \mathrm{M}$ cells were seeded into 96-well plates for overnight incubation, followed by pretreatment with $60 \mathrm{nM}$ PGRMC1-specific small interfering RNA (siRNA) or the corresponding GC content of scrambled (mis-matched) siRNA. Within $24 \mathrm{~h}$, the cells were treated with the indicated doxorubicin concentrations for $48 \mathrm{~h}$, followed by incubation with MTT for $4 \mathrm{~h}$. Dimethyl sulfoxide (DMSO) was then added and the plates shaken for 20 min followed by absorbance measurement at $540 \mathrm{~nm}$. Values were normalized against the untreated samples and are the average of four independent measurements \pm SD. $* P<0.05 ; * * P<0.01 ; * * * P<0.001$ vs. scrambled siRNA-transfected control cells. b Effect of PGRMC1 overexpression on the viability of the sensitive MES-SA cells. Values were normalized against the untreated samples and are the average of four

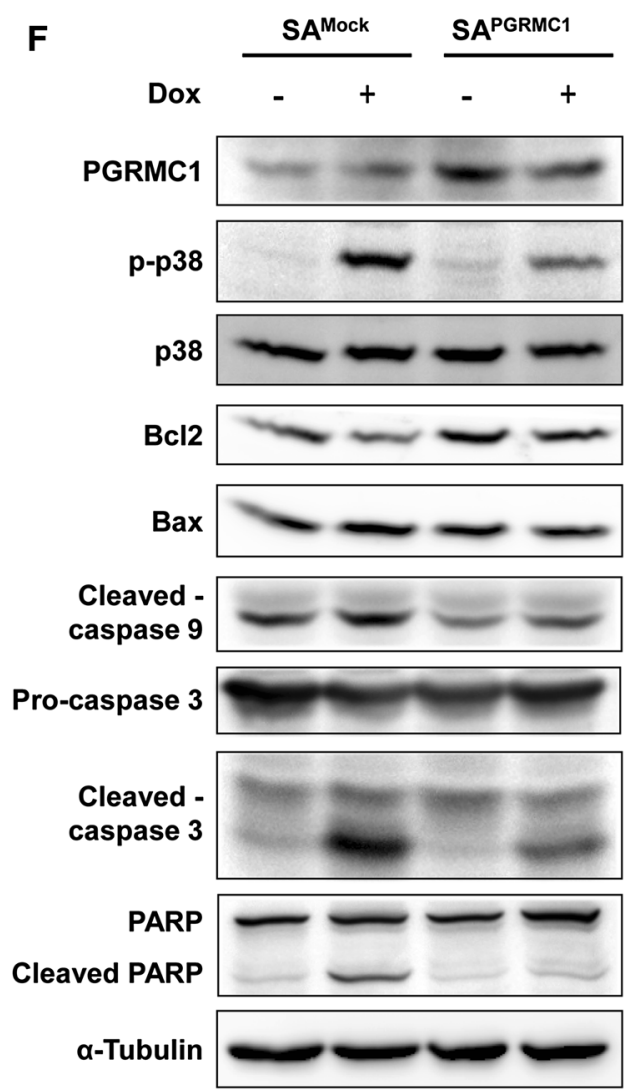

independent measurements \pm SD. $* P<0.05 ; * * P<0.01 ; * * * P<$ 0.001 vs. empty vector-transfected control cells $\left(M E S-S A{ }^{M o c k}\right)$. c Flow cytometry analysis of apoptosis using annexin $\mathrm{V}$ and propidium iodide (PI) staining. PGRMC1-silenced/scrambled siRNA-pretreated MES-SA, MES-SA/DxR-2 $\mu \mathrm{M}$, and MES-SA/ DxR-8 $\mu \mathrm{M}$ cells were treated with $1 \times$ half-maximal inhibitory concentration $\left(\mathrm{IC}_{50}\right)$ of doxorubicin $(D o x)$ or left untreated for $48 \mathrm{~h}$ $(C T R L)$. After treatment, $10^{6}$ cells were incubated with Alexa Fluor 488-conjugated annexin $\mathrm{V}$ and PI containing $1 \times$ binding buffer at room temperature for $15 \mathrm{~min}$. The stained cells were analyzed by flow cytometry. d Effect of doxorubicin on apoptosis in the control MESSA and PGRMC1-overexpressing MES-SA cells. Annexin V is presented on the $x$-axis as FL1-A, and PI is presented on the $y$-axis as FL2-A. The lower right quadrant indicates the percentage of early apoptotic cells (annexin V-positive cells), upper right quadrant indicates the percentage of late apoptotic cells (annexin V-positive and PI-positive cells). e, f Immunoblotting assay of indicated apoptotic factors with and without $0.5 \times \mathrm{IC}_{50}$ of doxorubicin for $24 \mathrm{~h}$ in the PGRMC1-silenced MES-SA/DxR-8 $\mu \mathrm{M}$ cells and PGRMC1-overexpressing MES-SA cells, respectively 